\title{
DOMAIN INVARIANCE IN INFINITE-DIMENSIONAL LINEAR SPACES
}

\author{
JAN VAN MILL
}

\begin{abstract}
Let $X$ be an infinite-dimensional locally convex linear space. It is known that $X$ is homeomorphic to a subspace of $X$ which is not open. We prove that every Banach space $B$ contains a dense linear subspace $L$ with the following property: If $U \subseteq L$ is open and if $f: U \rightarrow L$ is continuous and injective, then there exists a dense open $V \subseteq U$ such that (a) $f(V)$ is open in $L$, and (b) $f: V \rightarrow f(V)$ is a homeomorphism. As a consequence, $L$ and $L \times \mathbf{R}$ are not homeomorphic.
\end{abstract}

1. Introduction. The Brouwer invariance of domain property for Euclidean spaces implies that, for open $U \subseteq \mathbf{R}^{n}$, every injective map $g: U \rightarrow \mathbf{R}^{n}$ is an open imbedding [2]. It is well known that this property does not hold for infinitedimensional linear spaces. Indeed, for any infinite-dimensional normed linear space $Y$ we have the following examples.

EXAMPLE 1 [1, III, THEOREM 6.3]. There exists a homeomorphism $h: Y \rightarrow$ $h(Y)$ onto a nonopen subset of $Y$.

PROOF. Let $h_{0}: S \rightarrow H$ be any homemorphism from the unit sphere $S$ onto a closed hyperplane $H$. Then for any $y \in Y \backslash H, h_{0}$ may be extended to a homeomorphism $h$ of $Y$ onto the nonopen set $(H+(-\infty, 1) \cdot y) \cup\{y\}$ with $h(0)=y$.

EXAMPle 2 (D. W. CURTIS). There exists a bijective map $g: Y \rightarrow Y$ such that $g \mid Y \backslash K$ is not a homeomorphism for any compact $K \subseteq Y$.

PROOF. Since the unit sphere $S$ is noncompact, there exists a map $\lambda: S \rightarrow(0,1]$ such that inf $\lambda(S)=0$. Define $f: Y \rightarrow Y$ by the formulas

$$
\left\{\begin{array}{l}
f(y)=\lambda(y /\|y\|) \cdot y, \quad y \neq 0, \\
f(0)=0 .
\end{array}\right.
$$

Clearly, $f$ is a bijective map of $Y$, but is not a homeomorphism since $0 \notin \operatorname{int} f(B)$ for any bounded set $B$. Note that $f \mid Y \backslash\{0\}$ is a homeomorphism.

Using copies of $f$ on a discrete sequence of open balls in $Y$, we may construct a bijective map $g: Y \rightarrow Y$ such that for any compact $K \subseteq Y, g \mid Y \backslash K$ is not a homeomorphism. In fact, there exists an open $U \subseteq Y \backslash K$ such that for any compact $J \subseteq U, g(U \backslash J)$ is nonopen. However, there is a dense open $V \subseteq Y$ such that $g \mid V$ is an open imbedding.

The constructions in this paper show the existence of infinite-dimensional normed linear spaces $Y$ with the property that, for every injective map $g: U \rightarrow Y$ with open

Received by the editors June 18, 1986.

1980 Mathematics Subject Classification (1985 Revision). Primary 57N17.

Key words and phrases. Normed linear space, domain invariance.

Research partially supported by NSF grant DMS-8606113. This paper was written during a visit to Wesleyan University in June, 1986. The author is pleased to thank the Department of Mathematics at Wesleyan University for generous hospitality and support. 
domain, there is a dense open $V \subseteq U$ such that $g \mid V$ is an open imbedding. This property easily implies that $Y$ is not homeomorphic to $Y \times \mathbf{R}$. In fact, if $Y$ is homeomorphic to $Y \times Z$ then $Z$ is a single point.

Our results generalize Pol [7] and answer Question LS12 in Geoghegan [3]. I am greatly indebted to Doug Curtis for many helpful comments. He reworked and significantly simplified my original write-up when presenting the results to a seminar at LSU.

2. Special linear subspaces. Let $X$ be a separable metric linear space and let $\mathcal{K}(X)$ denote the collection of all homeomorphisms $h: K_{1} \rightarrow K_{2}$ between disjoint Cantor sets in $X$ such that $K_{1} \cup K_{2}$ is a linearly independent set.

2.1. THEOREM. Every separable metric linear space $X$ has a linear subspace $Y$ with the following property:

(*) for each $h \in \mathcal{K}(X)$, there exists $x \in \operatorname{dom} h$ such that $x \in Y$ but $h(x) \notin Y$.

PrOOF. It is easy to see that the collection $\mathcal{K}(X)$ has size at most $\mathfrak{c}$. Let $<$ be a well-ordering of $\mathcal{K}=\mathcal{K}(X)$ such that for each $h \in \mathcal{K}$, the section $\{g \in \mathcal{K}: g<h\}$ has size less than $c$.

We show by transfinite induction that for all $h \in \mathcal{K}$, there exist linear subspaces $Y_{h}$ and $Z_{h}$ in $X$ satisfying the following conditions.

(1) $Y_{h} \cap Z_{h}=\{0\}$;

(2) for $g<h, Y_{g} \subseteq Y_{h}$ and $Z_{g} \subseteq Z_{h}$;

(3) all $Y_{h}$ and $Z_{h}$ have algebraic dimension less than or equal to the cardinality of the section $\{g \in \mathcal{K}: g<h\}$; and

(4) there exists $x \in \operatorname{dom} h$ such that $x \in Y_{h}$ and $h(x) \in Z_{h}$.

Then $Y=\bigcup\left\{Y_{h}: h \in \mathcal{K}\right\}$ is a linear subspace with the required property (*).

For the first element $f \in \mathcal{K}$, we may take $Y_{f}=\operatorname{span}\{x\}$ and $Z_{f}=\operatorname{span}\{f(x)\}$ for any $x \in \operatorname{dom} f$. For $h \in \mathcal{K}$, suppose the spaces $Y_{g}$ and $Z_{g}$ have been constructed for $g<h$. Let $Y^{h}=\bigcup\left\{Y_{g}: g<h\right\}$ and $Z^{h}=\bigcup\left\{Z_{g}: g<h\right\}$. Then $Y^{h} \cap Z^{h}=\{0\}$, and $\operatorname{span}\left(Y^{h} \cup Z^{h}\right)$ has algebraic dimension less than $\mathfrak{c}$. Consider

$$
H=\left\{x \in \operatorname{dom} h: \operatorname{span}\left(\{x\} \cup Y^{h}\right) \cap \operatorname{span}\left(\{h(x)\} \cup Z^{h}\right) \neq\{0\}\right\} .
$$

We claim that $H$ has size less than c. For each $x \in H$, there exist scalars $\lambda(x)$ and $\mu(x)$ such that

$$
w(x)=\lambda(x) \cdot x+\mu(x) \cdot h(x) \in \operatorname{span}\left(Y^{h} \cup Z^{h}\right) \backslash\{0\} .
$$

Thus either $\lambda(x) \neq 0$ or $\mu(x) \neq 0$. To establish the claim on $H$, we show that the correspondence of $x \rightarrow w(x)$ is injective and has linearly independent range $\{w(x): x \in H\}$. Consider any finite, faithfully indexed subset $\left\{x_{1}, \ldots, x_{n}\right\}$ of $H$. Let $I=\left\{i: \lambda\left(x_{i}\right)=0\right\}$ and $J=\left\{i: \lambda\left(x_{i}\right) \neq 0\right\}$. Then $\operatorname{span}\left\{x_{i}, h\left(x_{i}\right)\right\}=$ $\operatorname{span}\left\{x_{i}, w\left(x_{i}\right)\right\}$ for $i \in I$, and $\operatorname{span}\left\{x_{i}, h\left(x_{i}\right)\right\}=\operatorname{span}\left\{h\left(x_{i}\right), w\left(x_{i}\right)\right\}$ for $i \in J$. Hence the set $\left\{x_{i}: i \in I\right\} \cup\left\{h\left(x_{i}\right): i \in J\right\} \cup\left\{w\left(x_{i}\right): 1 \leq i \leq n\right\}$ has the same span as the set $\left\{x_{i}: 1 \leq i \leq n\right\} \cup\left\{h\left(x_{i}\right): 1 \leq i \leq n\right\}$. The stipulated properties of $h \in \mathcal{K}$ imply that the latter set has size $2 n$ and is linearly independent. It follows that $\left\{w\left(x_{i}\right): 1 \leq i \leq n\right\}$ has size $n$ and in linearly independent. Since $\left\{x_{i}: 1 \leq i \leq n\right\} \subseteq H$ was arbitrary, this completes the argument for the claim on $H$. 
Since $H$ has size less than the size of the Cantor set dom $h$, there exists $x \in$ $\operatorname{dom} h \backslash H$, i.e.

$$
\operatorname{span}\left(\{x\} \cup Y^{h}\right) \cap \operatorname{span}\left(\{h(x)\} \cup Z^{h}\right)=\{0\} .
$$

The induction step is completed by setting $Y_{h}=\operatorname{span}\left(\{x\} \cup Y^{h}\right)$ and $Z_{h}=$ $\operatorname{span}\left(\{h(x)\} \cup Z^{h}\right)$.

3. Countable type for maps. A function $f: Y \rightarrow Y$ on linear space has countable type if there exists a countable set $Z$ in $Y$ such that for each $y \in Y, f(y) \in$ $\operatorname{span}(\{y\} \cup Z)$.

If each map $f: Y \rightarrow Y$ on a topological linear space has countable type, we say that $Y$ has countable type for maps.

Observe that if $Y$ is $\aleph_{0}$-dimensional, i.e. $Y$ has a Hamel basis of cardinality at most $\aleph_{0}$, then $Y$ has countable type for maps. The aim of this section is to show that there exist normed linear spaces having countable type for maps which are not $\aleph_{0}$-dimensional.

3.1. Definition. Let $X$ be a linear space, and $n$ a positive integer. A finite subset $\left\{x_{1}, \ldots, x_{k}\right\}$ of $X$ is said to be $n$-linearly essential if $\sum_{1}^{k} \lambda_{i} x_{i} \neq 0$ for all $\lambda_{i}$ such that $1 / n \leq\left|\lambda_{i}\right| \leq n$ for each $i$.

The following lemma is trivial; nonetheless it will be quite useful for establishing linear independence of a set which is constructed by "approximation" to a sequence of linearly independent sets.

3.2. LEMMA. (1) A subset $K$ of a linear space is linearly independent if and only if each finite subset of $K$ is n-linearly essential for each $n$.

(2) If $\left\{x_{1}, \ldots, x_{k}\right\}$ is an n-linearly essential subset of a metric linear space, there exists $\varepsilon>0$ such that, if $d\left(x_{i}, y_{i}\right)<\varepsilon$ or each $i$, then the set $\left\{y_{1}, \ldots, y_{k}\right\}$ is also $n$-linearly essential.

3.3. Definition. Let $X$ be a linear space, and $g: A \rightarrow X$ a function defined on a subset of $X$. A subset $P$ of $A$ is said to be $g$-independent if the following conditions are satisfied:

(i) $g \mid P$ is injective;

(ii) $P \cap g(P)=\varnothing$; and

(iii) $P \cup g(P)$ is linearly independent.

3.4. Proposition. Let $X$ be a metric linear space, and $g: A \rightarrow X$ a map defined on a separable, topologically complete subset. If $A$ contains an uncountable $g$-independent set, then $A$ contains a $g$-independent Cantor set.

ProOF. Let $d$ be a metric on $X$, and choose a complete metric $\rho$ on $A$. For each $x \in A$ and $\varepsilon>0$, let $B(x, \varepsilon)=\{a \in A: \rho(a, x) \leq \varepsilon\}$. Since each separable metric space is the union of a countable set and a perfect set (each point a limit point), the hypothesis implies that $A$ contains a perfect $g$-independent set $P$. Using finite disjoint unions of balls about points of $P$, we may construct a Cantor set $K$ in the complete space $A$ by the standard procedure; a little extra care will ensure that $K$ is $g$-independent. It suffices to describe the first two steps in the inductive construction.

Pick any $p_{1} \in P$. Since $g\left(p_{1}\right) \neq p_{1}$, there exists $0<\varepsilon_{1}<1$ such that $B\left(p_{1}, \varepsilon_{1}\right) \cap g\left(B\left(p_{1}, \varepsilon_{1}\right)\right)=\varnothing$. Let $B_{1}=B\left(p_{1}, \varepsilon_{1}\right)$. Since the set $\left\{p_{1}, g\left(p_{1}\right)\right\}$ is 
linearly independent, we may assume by Lemma $3.2(2)$ that $\varepsilon_{1}$ is sufficiently small so that, for any $F \subseteq B_{1} \cup g\left(B_{1}\right)$ such that $F$ contains at most a single point from each of $B_{1}$ and $g\left(B_{1}\right), F$ is 1-linearly essential. Let $K_{1}=B_{1}$.

Since $P$ is perfect, there exist distinct points $p_{1,0}$ and $p_{1,1}$ in $P \cap B_{1}$. Choose $0<\varepsilon_{2}<\frac{1}{2}$ such that, for $B_{1,0}=B\left(P_{1,0}, \varepsilon_{2}\right)$ and $B_{1,1}=B\left(p_{1,1}, \varepsilon_{2}\right)$, we have $B_{1,0} \cup B_{1,1} \subseteq B_{1}, B_{1,0} \cap B_{1,1}=\varnothing$, and $g\left(B_{1,0}\right) \cap g\left(B_{1,1}\right)=\varnothing$. Since the set $\left\{p_{1,0}, p_{1,1}, g\left(p_{1,0}\right), g\left(p_{1,1}\right)\right\}$ is linearly independent and has size 4 , we may also assume that $\varepsilon_{2}$ is small enough so that, for any $F \subseteq B_{1,0} \cup B_{1,1} \cup g\left(B_{1,0}\right) \cup g\left(B_{1,1}\right)$ such that $F$ contains at most a single point from each of $B_{1,0}, B_{1,1}, g\left(B_{1,0}\right)$, and $g\left(B_{1,1}\right), F$ is 2-linearly essential. Set $K_{2}=B_{1,0} \cup B_{1,1}$.

Continuing with this procedure in the standard manner, we obtain a nested sequence $\left(K_{n}\right)$ of closed sets in $A$. Let $K=\bigcap_{1}^{\infty} K_{n}$. The requirements of the type $B_{1,0} \cup B_{1,1} \subseteq B_{1}$ and $B_{1,0} \cap B_{1,1}=\varnothing$, together with the requirement that $\varepsilon_{n} \rightarrow 0$ and the fact that $\rho$ is a complete metric, show that $K$ is a Cantor set. The requirements of the type $g\left(B_{1,0}\right) \cap g\left(B_{1,1}\right)=\varnothing$ show that $g \mid K$ is injective. Since $g(K) \subseteq g\left(B_{1}\right)$ and $K \subseteq B_{1}, K \cap g(K)=\varnothing$. And finally, the $n$-linearly essential requirement at the $n$ th-stage of construction ensures that each finite subset of $K \cup g(K)$ is $n$-linearly essential for each $n$, hence $K \cup g(K)$ is linearly independent by Lemma $3.2(1)$. Thus $K$ is $g$-independent.

REMARK. If we assume only that $A$ contains an uncountable linearly independent set, the above construction shows that $A$ contains a linearly independent Cantor set.

3.5. Proposition. A function $f: Y \rightarrow Y$ on a linear space has countable type if and only if it satisfies the following conditions:

(i) every $f$-independent set is countable; and

(ii) for every countable set $P \subseteq Y$, there exists a countable set $\hat{P} \subseteq Y$ such that $f(\operatorname{span} P) \subseteq \operatorname{span} \hat{P}$.

ProOF. Suppose first that $f$ has countable type; let $Z$ be a countable subset of $Y$ such that $f(y) \in \operatorname{span}(\{y\} \cup Z)$ for each $y$. Let $T=\{y \in Y: f(y) \notin \operatorname{span}\{y\}\}$. Then for each $y \in T$, span $Z \cap \operatorname{span}\{y, f(y)\} \neq\{0\}$. Consider any $f$-independent set $A$. We have $A \subseteq T$, and for each $a \in A$ we may choose $s_{a} \in(\operatorname{span} Z \cap$ $\operatorname{span}\{a, f(a)\}) \backslash\{0\}$. Since $A$ is $f$-independent, the set $\left\{s_{a}: a \in A\right\}$ is a linearly independent subset of $\operatorname{span} Z$, and is therefore countable. It follows that $A$ is countable, and condition (i) is satisfied. Condition (ii) is clear, since $f(\operatorname{span} P) \subseteq$ $\operatorname{span}(P \cup Z)$ for every $P \subseteq Y$.

Conversely, assume conditions (i) and (ii). It is easily seen that in the collection of all $f$-independent subsets, partially ordered by inclusion, every chain has an upper bound. Thus there exists a maximal $f$-independent set $Q$, which by hypothesis is countable. If $Q=\varnothing$, then $f(y) \in \operatorname{span}\{y\}$ for each $y \neq 0$, and $f$ obviously has countable type. Otherwise, construct a tower $\left(P_{i}\right)$ of countable sets by taking $P_{1}=Q$, and $P_{n+1}=P_{n} \cup \hat{P}_{n}$ for $n \geq 1$. Take $Z=\bigcup_{1}^{\infty} P_{n}$. Then $Z$ is countable, $Q \subseteq Z$ and $f(\operatorname{span} Z) \subseteq \operatorname{span} Z$. We claim that $f(y) \in \operatorname{span}(\{y\} \cup Z)$ for each $y$. This is clear for $y \in \operatorname{span} Z$ so consider $y \notin \operatorname{span} Z$. The set $Q \cup\{y\}$ cannot be $f$-independent, and one of the following occurs:

(1) $f(y)=f(q)$ for some $q \in Q$. Then $f(y) \in \operatorname{span} Z$.

(2) $y=f(q)$ for some $q \in Q$. Then $y \in \operatorname{span} Z$. 
(3) $\{y, f(y)\} \cup Q \cup f(Q)$ is not linearly independent. in this case, either

$$
f(y) \in \operatorname{span}(\{y\} \cup Q \cup f(Q)) \subseteq \operatorname{span}(\{y\} \cup Z),
$$

or

$$
y \in \operatorname{span}(Q \cup f(Q)) \subseteq \operatorname{span} Z .
$$

Thus $f$ has countable type.

We now come to the main result in this section.

3.6. THEOREM. Let $X$ be an infinite-dimensional separable, complete metric linear space, and $Y$ a linear subspace with the property $(*)$ of Theorem 2.1 . Then

(1) $Y$ is dense in $X$;

(2) $Y$ is Baire space; and

(3) $Y$ has countable type for maps.

ProOF. We show first that $Y$ intersects every dense $G_{\delta}$-subset of $X$. Every such set $A$ must contain an uncountable linearly independent set, since otherwise span $A$ is an $\aleph_{0}$-dimensional linear subspace, and $X=\operatorname{span} A \cup(X \backslash A)$ would be first category. Then $A$ contains a linearly independent Cantor set $K$ (see the remark at the end of proof of Proposition 3.4; see also [6]). Let $h: K_{1} \rightarrow K_{2}$ be any homeomorphism between disjoint Cantor subsets of $K$. Then $h \in \mathcal{K}(X)$, so $Y \cap A \supseteq Y \cap K_{1} \neq \varnothing$.

In particular, $\bar{Y}$ must have nonempty interior in $X$, which implies that $\bar{Y}=X$. Further, the fact that $Y$ intersects every dense $G_{\delta}$-subset of $X$ means that $Y$ is second category, which implies that $Y$ is a Baire space.

We now show that every map $f: Y \rightarrow Y$ has countable type. Since $X$ is complete, $f$ extends to a map $g: A \rightarrow X$ for some $G_{\delta}$-subset $A$ of $X$. Suppose that $A$ contains an uncountble $g$-independent set. By Proposition $3.4, A$ contains a $g$ independent Cantor set $K$. Then $g \mid K$ is a member of the collection $K(X)$, and by hypothesis there exists $x \in K \cap Y$ such that $g(x) \notin Y$. But this contradicts the fact that $g(x)=f(x) \in Y$. Thus every $g$-independent subset of $A$ is countable, and in particular, every $f$-independent set is countable. Therefore, condition (i) of Proposition 3.5 is satisfied.

It remains to verify condition (ii). For any countable set $P \subseteq Y$, span $P$ is $\sigma$-compact, and $f(\operatorname{span} P)$ is $\sigma$-compact. If some compactum in $Y$ contains an uncountable linearly independent set, then $Y$ contains a linearly independent Cantor set, but this contradicts property $(*)$. (If $K \subseteq Y$ is a linearly independent Cantor set, let $h: K_{1} \rightarrow K_{2}$ be any homeomorphism between Cantor subsets of $K$; by assumption there exists $x \in K_{1}$ such that $h(x) \in Y$, which is a contradiction.) Thus each compactum in $Y$ lies in an $\aleph_{0}$-dimensional linear subspace, and $f(\operatorname{span} P) \subseteq \operatorname{span} \hat{P}$ for some countable set $\hat{P}$. This completes the proof that $Y$ has countable type for maps, Proposition 3.5.

4. Domain invariance. Let $Y$ be a space. Suppose that, for every injective map $g: U \rightarrow Y$ with domain an open subset of $Y$, there exists a nonempty open $V \subseteq U$ such that $g \mid V$ is an open imbedding in $Y$. Then we say that $Y$ has restricted domain invariance.

We may suppose that $V$ is dense in $U$, since the condition can be applied to every restriction $g \mid W$ to an open $W \subseteq U$. 
For $Y$ a normed linear space, it suffices to verify the condition for every injective map $f: Y \rightarrow Y$, since there is an open imbedding of $Y$ into every nonempty open subset.

The aim of this section to prove that every normed linear space with the Baire property and with countable type for maps has restricted domain invariance.

4.1. Proposition. Let $Y$ be a normed linear space, $F$ a finite-dimensional linear subspace, and $V \subseteq Y \backslash F$ an open subset. Let $\lambda: V \rightarrow(0, \infty)$ and $\alpha: V \rightarrow F$ be maps such that the map $f: V \rightarrow Y$ defined by $f(V)=\lambda(v) \cdot v+\alpha(v)$ is injective. Then $f$ is an open imbedding.

PROOF. Since the restriction $f \mid W$ to any open $W \subseteq V$ is a map with the same properties, it suffices to show that $f(V)$ is open.

Consider an arbitrary point $p \in V$. We may assume that $\lambda(p)=1$ and $\alpha(p)=0$, thus $f(p)=p$. Let $E=\operatorname{span}(\{p\} \cup F)$. Note that $f(E \cap V) \subseteq E$. Choose $\delta>0$ and a compact neighborhood $B$ of 0 in $F$ such that $D=[1-\delta, 1+\delta] \cdot p+B \subseteq V$. Then $D$ is a compact neighborhood of $p$ in $E$. Since $f$ is injective and $E$ is finite dimensional, $f \mid D: D \rightarrow E$ is an imbedding and $f(D)$ is a neighborhood of $f(p)=p$ in $E[2]$. Thus $p$ is a stable point of $f(D)$, and any map $\tilde{f}: D \rightarrow E$ which is sufficiently close to $f \mid D$ must cover $p$.

For each $v \in V$, let $E_{v}=\operatorname{span}(\{v\} \cup F)$, and define the homeomorphism $h_{v}: E \rightarrow$ $E_{v}$ by

$$
h_{v}(t \cdot p+s)=t \cdot v+s
$$

for $t \in(-\infty, \infty)$ and $s \in F$. Let $D_{v}=h_{v}(D)=[1-\delta, 1+\delta] \cdot v+B$. Then for all $v$ near $p, D_{v} \subseteq V$ and $f\left(D_{v}\right) \subseteq E_{v}$. Define a map $\tilde{f}_{v}: D \rightarrow E$ by $\tilde{f}_{v}=h_{v}^{-1} \circ f \circ h_{v} \mid D$. Then $\tilde{f}_{v} \rightarrow f \mid D$ as $v \rightarrow p$. Hence for all $v$ sufficiently close to $p, p \in \tilde{f}_{v}(D)$, which means that $v \in f\left(D_{v}\right)$. Thus $f(V)$ is a neighborhood of $p=f(p)$.

We now come to the main result in this section.

4.2. THEOREM. Let $Y$ be a normed linear space with the Baire property and with countable type for maps. Then $Y$ has restricted domain invariance.

ProOF. We may assume $Y$ is infinite dimensional [2]. By the remarks at the beginning of this section, we need only consider an injective map $f: Y \rightarrow Y$. Let $Z \subseteq Y$ be a countable set such that $f(y) \in \operatorname{span}(\{y\} \cup Z)$ for each $y$. There is a tower of compacta $\left(A_{n}\right)$ such that $\operatorname{span} A_{n}$ is finite dimensional for each $n$, and $\bigcup_{1}^{\infty} A_{n}=\operatorname{span} Z$. For each $n$, set

$$
Y_{n}=\left\{y \in Y: \text { for some } \lambda \in[-n, n], f(y)-\lambda \cdot y \in A_{n}\right\} \text {. }
$$

By compactness of $[-n, n]$ and $A_{n}$, each $Y_{n}$ is closed in $Y$. Since $\bigcup_{1}^{\infty} Y_{n}=Y$, and $Y$ is a Baire space, some $Y_{n}$ has nonempty interior. Since span $A_{n}$ is finite dimensional, there exists a nonempty open set $W \subseteq Y_{n} \backslash \operatorname{span} A_{n}$. Then for each $w \in W$, there is a unique $\lambda(w) \in[-n, n]$ such that $f(w)-\lambda(w) \cdot w \in A_{n}$ (otherwise, $W \cap \operatorname{span} A_{n} \neq \varnothing$ ). Furthermore, the compactness of $[-n, n]$ and $A_{n}$, and the continuity of $f$, show that the assignment $w \rightarrow \lambda(w)$ is continuous. Let $\lambda: W \rightarrow[-n, n]$ denote this map, and let $\alpha: W \rightarrow A_{n}$ denote the map defined by $\alpha(w)=f(w)-\lambda(w) \cdot w$. If $\lambda(W)=\{0\}$, we have $f(W) \subseteq A_{n}$. But this is impossible, since $f$ is injective, the open set $W$ contains cells of all dimensions, and $A_{n}$ is finite dimensional. Thus, there exists a nonempty open $V \subseteq W$ with either $\lambda(V) \subseteq[-n, 0)$ or $\lambda(V) \subseteq(0, n]$. In the latter 
case an, application of Proposition 4.1, with $F=\operatorname{span} A_{n}$, shows that $f \mid V$ is an open imbedding. And in the former case, the map $(-1) \cdot f \mid V$ is an open imbedding, hence so is $f \mid V$. This completes the proof of restricted domain invariance for $Y$.

4.3. COROLLARY. Every separable Banach space contains a dense linear subspace $Y$ such that:

(1) $Y$ is a Baire space; and

(2) $Y$ has restricted domain invariance.

ProOF. Combine Theorems 2.1, 3.6, and 4.2.

4.4. COROllary to COROLlARY. Every separable Banach space contains a dense linear subspace $Y$ such that for all closed linear subspaces $E$ and $F$ of $Y$ of finite codimension the following statements are equivalent:

(1) $E$ and $F$ are homeomorphic;

(2) $E$ and $F$ are linearly homeomorphic; and

(3) $\operatorname{codim} E=\operatorname{codim} F$.

Proof. Let $Y$ be as in Corollary 4.3 and let $E$ and $F$ be closed linear subspaces of $Y$ of finite codimension.

That (3) implies (2) follows from [1, III, Proposition 1.5]. Clearly, (2) implies (1). suppose that $E$ and $F$ are homeomorphic and that $n=\operatorname{codim} E-\operatorname{codim} F>0$. By [1, III, Proposition 1.5], $E \times \mathbf{R}^{n}$ and $F$ are linearly homeomorphic. Consequently, $E$ and $E \times \mathbf{R}^{n}$ are homeomorphic. Since $Y$ has countable type for maps, every closed linear subspace of $Y$ has the same property. From Theorem 4.2 we therefore conclude that $E$ has restricted domain invariance, which contradicts $E$ being homeomorphic to $E \times \mathbf{R}^{n}$.

5. Further applications and remarks. By Corollary 4.3, there exists an infinite-dimensional pre-Hilbert space $L$ with restricted domain invariance. Consequently, $L \neq L \times E$ for any nondegenerate space $E$. This result generalizes Pol [7] and answers Question LS12 in Geoghegan [3].

In fact, there exists an infinite-dimensional pre-Hilbert space $L$ such that $L \times F \not$ $L \times G$ for any linear spaces $F$ and $G$ with different dimensions (finite or $\infty$ ). Let $Y$ be an infinite-dimensional pre-Hilbert space which is a Baire space and which has countable type for maps. It is easily seen that every closed linear subspace in $Y$ inherits these properties, and therefore has restricted domain invariance. Take $L$ to be an infinite-dimensional closed linear subspace of $Y$ with infinite codimension. Suppose $L \times F \approx L \times G$ for certain linear spaces $F$ and $G$ with $0 \leq \operatorname{dim} F<$ $\operatorname{dim} G \leq \infty$. Consider a closed linear subspace $M$ in $Y$ containing $L$, such that the codimension of $L$ in $M$ equals $\operatorname{dim} F$. Since $M$ is closed in $Y, M$ has restricted domain invariance. We have $M \approx L \times F$, and $G \approx F \times E$ for some nondegenerate space $E$. Then the supposition that $L \times F \approx L \times G$ implies $M \approx M \times E$, a contradiction.

A linear space $Y$ is said to have few operators if every bounded linear operator $A: Y \rightarrow Y$ has the form $A=\lambda I+B$ for some scalar $\lambda$ and some operator $B$ with finite-dimensional range. In [5], I shall prove that every Baire linear space with countable type for maps has few operators.

The method used in the proofs of Theorems 2.1 and 3.6 was inspired by Sierpiński [8]. It improves the method used in [4] and can be used to construct a topological 
group $G$ such that every continuous function $f: G \rightarrow G$ is either constant or a translation. This answers a question in [4].

\section{REFERENCES}

1. C. Bessaga and A. Pełczyński, Selected topics in infinite-dimensional topology, PWN, Warsaw, 1975.

2. L. E. J. Brouwer, Invarianz des n-dimensionalen Gebiets, Math. Ann. 71 (1912), 305-313; 72 (1912), 55-56.

3. R. Geoghegan (ed.), Open problems in infinite-dimensional topology, Topology Proc. 4 (1979), 287-338.

4. J. van Mill, A topological group having no homeomorphisms other than translations, Trans. Amer. Math. Soc. 280 (1983), 491-498.

5. __ Infinite-dimensional normed linear spaces and domain invariance, Mathematics and Computer Science II, CWI Monographs 4, North-Holland, 1986, pp. 105-110.

6. J. van Mill and R. Pol, The Baire Category Theorem in products of linear spaces and topological groups, Topology Appl. 22 (1986), 267-282.

7. R. Pol, An infinite-dimensional pre-Hilbert space not homeomorphic to its own square, Proc. Amer. Math. Soc. 90 (1984), 450-454.

8. W. Sierpiński, Sur un probleme concernant les types de dimensions, Fund. Math. 19 (1932), 65-71.

SubFaCUlteit Wiskunde EN INFORMatica, VRIJe UNiversiteit, De BoelelaAN 1081, 1081 HV AMSTERDAM, THE NETHERLANDS

Mathematisch InstituUt, Universiteit VAN Amsterdam, RoetersstraAt 15, 1018 WB AMSTERDAM, THE NETHERLANDS 